\title{
Eurolung risk score is associated with long-term survival after curative resection for lung cancer
}

Alessandro Brunelli, MD, Nilanjan Chaudhuri, MD, Manos Kefaloyannis, MD, Richard Milton, MD, Cecilia Pompili, MD, PhD, Peter Tcherveniakov, MD, and Kostas Papagiannopoulos, MD

\section{ABSTRACT}

Objective: The study objective was to verify whether the Eurolung score was associated with long-term prognosis after lung cancer resection.

Methods: A total of 1359 consecutive patients undergoing anatomic lung resection (1136 lobectomies, 103 pneumonectomies, 120 segmentectomies) (2014-2018) were analyzed. The parsimonious aggregate Eurolung2 score was calculated for each patient. Median follow-up was 802 days. Survival distribution was estimated by the Kaplan-Meier method. Cox proportional hazard regression and competing risk regression analyses were used to assess the independent association of Eurolung with overall and disease-specific survival.

Results: Patients were grouped into 4 classes according to their Eurolung scores (A $0-2.5, B 3-5, C 5.5-6.5, D 7-11.5)$. Most patients were in class A (52\%) and B (33\%), 8\% were in class $C$, and $7 \%$ were in class D. Five-year overall survival decreased across the categories (A: $75 \%$; B: $52 \%$; C: $29 \%$; D: $27 \%$, log rank $P<.0001$ ). The score stratified the 3 -year overall survival in patients with $\mathrm{pT} 1(P<.0001)$ or $\mathrm{PT}>1$ $(P<.0001)$. In addition, the different classes were associated with incremental risk of long-term overall mortality in patients with $\mathrm{pNo}(P<.0001)$ and positive no$\operatorname{des}(P=.0005)$. Cox proportional hazard regression and competing regression analyses showed that Eurolung aggregate score remained significantly associated with overall (hazard ratio, 1.19; $P<.0001$ ) and disease-specific survival after adjusting for PT and pN stage (hazard ratio, 1.09; $P=.005$ ).

Conclusions: Eurolung aggregate score was associated with long-term survival after curative resection for cancer. This information may be valuable to inform the shared decision-making process and the multidisciplinary team discussion assisting in the selection of the most appropriate curative treatment in high-risk patients. ( $\mathrm{J}$ Thorac Cardiovasc Surg 2021;161:776-86)

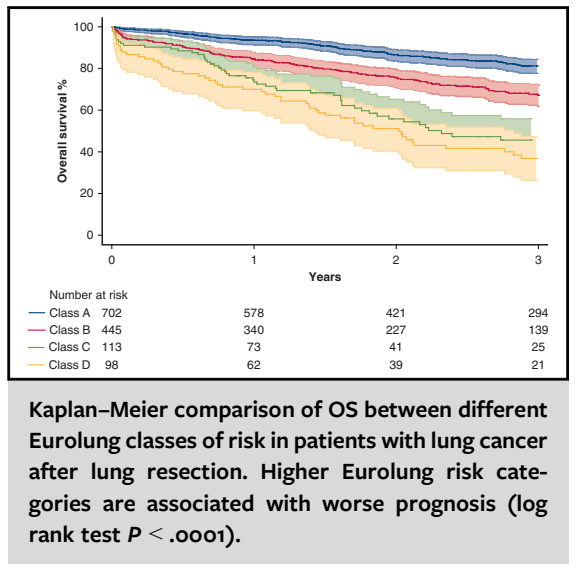

CENTRAL MESSAGE

Eurolung can assist the shared decision-making process and the multidisciplinary team discussion to select the most appropriate curative treatment in high-risk patients.

\section{PERSPECTIVE}

Eurolung score was associated with long-term overall and DSS after lung cancer resection independent of $\mathrm{pT}$ and $\mathrm{pN}$ stage. This finding highlights the importance of applying mixed models incorporating not only oncologic but also patient- and treatment-related characteristics to stratify prognosis after curative treatment for lung cancer

See Commentaries on pages 787 and 788.
From the Department of Thoracic Surgery, St James's University Hospital Bexley Wing, Leeds, United Kingdom.

Read at the 100th Annual Meeting of The American Association for Thoracic Surgery: A Virtual Learning Experience, May 22-23, 2020.

Received for publication April 30, 2020; revisions received June 9, 2020; accepted for publication June 11, 2020; available ahead of print Aug 24, 2020.

Address for reprints: Alessandro Brunelli, MD, Department of Thoracic Surgery, St James's University Hospital Bexley Wing, Beckett St, Leeds LS9 7TF, United Kingdom (E-mail: alexit_2000@yahoo.com).

$0022-5223 / \$ 36.00$

Copyright (c) 2020 by The American Association for Thoracic Surgery

https://doi.org/10.1016/j.jtcvs.2020.06.151
The Eurolung is a model developed from the European Society of Thoracic Surgeons (ESTS) database to stratify the risk of immediate postoperative mortality based on patient- and surgical-related characteristics. ${ }^{1}$ Recent evidence has shown that different physiologic and patient-related factors may influence not only perioperative

To view the AATS Annual Meeting Webcast, see the URL next to the webcast thumbnail. 


\section{Abbreviations and Acronyms \\ BMI = body mass index \\ CI $=$ confidence interval \\ DSS $=$ disease-specific survival \\ ESTS = European Society of Thoracic Surgeons \\ OS = overall survival \\ ppoFEV1 $=$ predicted postoperative forced \\ expiratory volume in 1 second}

outcomes but also long-term survival after curative treatment for cancer. ${ }^{2-6}$ Because the Eurolung model includes nononcologic factors potentially associated with prognosis, it may represent a valuable tool to estimate not only the immediate risk of death after surgery but also the long-term mortality after curative resection. The objective of this study was to verify whether the Eurolung aggregate score was associated with overall and disease-specific survival (DSS) after lung cancer resection.

\section{PATIENTS AND METHODS}

This is a retrospective single-center analysis performed on prospectively collected data. A total of 1359 consecutive patients undergoing anatomic lung resection for non-small cell lung cancer (1136 lobectomies, 103 pneumonectomies, 120 segmentectomies) from April 2014 to December 2018 were analyzed. The study was reviewed by the Research and Innovation Department of the hospital and classified as service evaluation not requiring review by an NHS Research Ethics Committee.

All patients were selected for operation after discussion in a multidisciplinary tumor board. A patient was deemed unfit for surgery in case of a predicted postoperative forced expiratory volume in 1 second (ppoFEV1) and predicted postoperative carbon monoxide lung diffusion capacity less than $30 \%$ of predicted associated with VO2max less than $10 \mathrm{~mL} / \mathrm{kg} /$ min, according to published guidelines. ${ }^{7}$

Preoperative staging was performed using systematic positron emission tomography computed tomography scan. Endobronchial ultrasound or endoscopic ultrasound nodal sampling was performed in case of positron emission tomography avid hilar or mediastinal lymph nodes. All patients were referred to surgeons after discussion at a multidisciplinary meeting.

All operations were performed by qualified thoracic surgeons, and patients were cared for in a dedicated thoracic surgery unit after surgery. Only patients with pathologically staged $\mathrm{R} 0$ resections were included in the analysis. A systematic lymph node dissection was performed in all patients. Patients were staged according to the 8th edition of the TNM staging system.

\section{Statistical Analysis}

Numeric variables are presented as means and standard deviations, and categoric variables are presented as count and percentages. For the purpose of this study, the parsimonious aggregate Eurolung2 score was calculated for each patient. The parsimonious Eurolung2 model is a simplified and updated version of the Eurolung model. ${ }^{1}$ It was recently developed from a population of more than 85,000 patients registered in the ESTS database and includes the following factors: age, ppoFEV1, body mass index (BMI), gender, open surgery (as opposed to video-assisted thoracoscopic surgery), and pneumonectomy (as opposed to lesser resections). All variables were complete with the exception of BMI and ppoFEV1, which had less than $5 \%$ of missing data. Missing values were replaced by multiple imputation. The aggregate Eurolung is a derived score that was created by categorizing the numeric variables in the model and after weighting the individual factors. ${ }^{1}$ It is obtained by summing the points generated by the following variables: age more than 70 years 1 point, ppoFEV1 less than $70 \% 1$ point, open surgery (as opposed to minimally invasive surgery) 2.5 points, BMI less than 18.52 .5 points, male sex 2.5 points, pneumonectomy (as opposed to lesser resection) 3 points. For the purpose of this study, patients were grouped according to the risk classes proposed in the original study, ${ }^{1}$ with the exception of patients with a score of 7 points or higher were grouped into a single class (class D) for numerosity reason. As a consequence, the patients in this study were categorized into 4 risk classes according to their Eurolung scores (Class A: 0-2.5, class B: 3-5, class C: 5.5-6.5 and class D: 7-11.5).

Follow-up information (including date and cause of death) was obtained by data retrieved from the centralized electronic clinical information system of the hospital, which records the date of death and any medical treatment or access to care including occurred in other regional Hospitals. All patients were followed up through August 2019. Median follow-up was 802 days (interquartile range, 396-1297). No patient was lost at followup. Survival was defined as the interval between surgery to death or last contact. Overall survival (OS) was measured from the date of surgery to

TABLE 1. Baseline characteristics of the patients included in the study (no. 1359)

\begin{tabular}{lc}
\hline \multicolumn{1}{c}{ Variables } \\
\hline Age & $67.8(10.4)$ \\
\hline Age $>70$ y $(\mathrm{n}, \%)$ & $625(46)$ \\
\hline Sex male $(\mathrm{n}, \%)$ & $643(47)$ \\
BMI $\left(\mathrm{kg} / \mathrm{m}^{2}\right)$ & $27.0(5.5)$ \\
\hline BMI $<18.5 \mathrm{~kg} / \mathrm{m}^{2}(\mathrm{n}, \%)$ & $38(2.8)$ \\
PS $>1(\mathrm{n}, \%)$ & $122(9.0)$ \\
\hline FEV1\% & $88.4(22.1)$ \\
ppoFEV1 $<70 \%(\mathrm{n}, \%)$ & $612(45)$ \\
\hline DLCO \% & $72.6(18.4)$ \\
ppoDLCO \% & $58.2(16.6)$ \\
CAD (n, \%) & $205(15)$ \\
CVD (n, \%) & $82(6.0)$ \\
\hline CKD (n, \%) & $30(2.2)$ \\
\hline Open access (as opposed to MITS) (n, \%) & $316(23)$ \\
\hline Pneumonectomies (n, \%) & $103(7.6)$ \\
\hline Lobectomies (n, \%) & $1136(83.6)$ \\
\hline Segmentectomies (n, \%) & $120(8.8)$ \\
\hline Histology (n, \%) & Squamous 453(33) \\
& Adenocarcinoma $731(54)$ \\
\hline & Others $175(13)$ \\
\hline
\end{tabular}

Results are expressed a means and standard deviations for numeric variables and as count and percentages for categoric variables. $B M I$, Body mass index; $P S$, performance score; FEVI, forced expiratory volume in 1 second; ppoFEVI, predicted postoperative forced expiratory volume in 1 second; $D L C O$, carbon monoxide lung diffusion capacity; $p p o D L C O$, predicted postoperative carbon monoxide lung diffusion capacity; $C A D$, coronary artery disease; $C V D$, cerebrovascular disease; $C K D$, chronic kidney disease; MITS, minimally invasive thoracic surgery. 
the date of death and censored at the date of last follow-up for survivors. DSS was measured from the date of surgery to the date of death from lung cancer and censored at the date of last follow-up for survivors or the date a patient died of causes other than lung cancer. Survival distribution was estimated by the Kaplan-Meier method and compared between groups using the log-rank test.

A Cox proportional hazard regression was used to assess the association of Eurolung with OS adjusting for other prognostic factors such as pT and pN stage, predicted postoperative carbon monoxide lung diffusion capacitypredicted postoperative carbon monoxide lung diffusion capacity, Eastern Cooperative Oncology Group performance score, and other comorbidities (coronary artery disease, cerebrovascular disease, diabetes, chronic kidney disease). Comorbidities were defined according to the joint European Society of Thoracic Surgery/Society of Thoracic Surgeons definitions. ${ }^{8}$

A competing regression analysis adjusting for $\mathrm{pT}, \mathrm{pN}$ stage, and patientrelated variables was used to test the independent association of Eurolung with DSS where the competing event was considered death from cancer other than lung cancer or death from causes other than cancer. The association between variables and hazard of lung cancer death was estimated using the Fine and Gray competing risk model. ${ }^{9}$ All tests were performed on Stata 15.1 statistical software (Stata Corp LP, College Station, Tex).

\section{RESULTS}

Table 1 reports the baseline characteristics of the patients included in this study. A total of 55 patients died within 30 days from operation (4\%). Three-year OS was $72 \%$ (95\% confidence interval [CI], 69-75), 42\% (95\% CI, $32-52)$, and $81 \%$ (95\% CI, 70-88) for lobectomies, pneumonectomies, and segmentectomies. There were 998 patients alive at the time of last follow-up. A total of 449 patients were alive at more than 3 years after surgery.
The median Eurolung score was 2.5 (interquartile range, 1-4.5) and ranged from 0 to 11.5 points. Most patients were in class $\mathrm{A}(52 \%)$ and $\mathrm{B}(33 \%), 8 \%$ were in class $\mathrm{C}$, and $7 \%$ were in class $\mathrm{D}$. There was an incremental 30-day mortality rate across the Eurolung categories (A: $0.9 \%, \mathrm{~B}: 5.4 \%, \mathrm{C}$ : $9.7 \%$, and D: $14 \%, P<.0001$ ).

Three-year OS decreased across the Eurolung classes (A: 78\% (95\% CI, 74-82); B: $61 \%$ (95\% CI, 55-66); C: $40 \%$ (95\% CI, 29-50); D: 36\% (95\% CI, 29-44), log rank $P<.0001$ (Figure 1). Pairwise comparisons between Eurolung classes (after Bonferroni adjustment for multiple comparisons) showed that there was a significantly $(P<.008)$ higher incremental risk of death from all causes by increasing class of risk with the exception of classes $\mathrm{C}$ and $\mathrm{D}$, which had similar OS.

Likewise, 3-year DSS decreased across the Eurolung classes (A: 91\% (95\% CI, 88-93); B: 85\% (95\% CI, 8089); C: $63 \%$ (95\% CI, 50-74); D: 65\% (95\% CI, 50-77), $\log \operatorname{rank} P<.0001)$. Pairwise comparisons between Eurolung classes (after Bonferroni adjustment for multiple comparisons) showed that there was a significantly $(P<.008)$ higher incremental risk of death from lung cancer by increasing class of risk with the exception of classes $\mathrm{C}$ and $\mathrm{D}$, which had similar DSS.

The score stratified well the 3-year OS in patients with pT1 $(P<.0001)$ or pT greater than $1(P<.0001)$ (Figure 2, $A$ and $B$ ). In addition, the different classes were associated with incremental risk of long-term mortality in

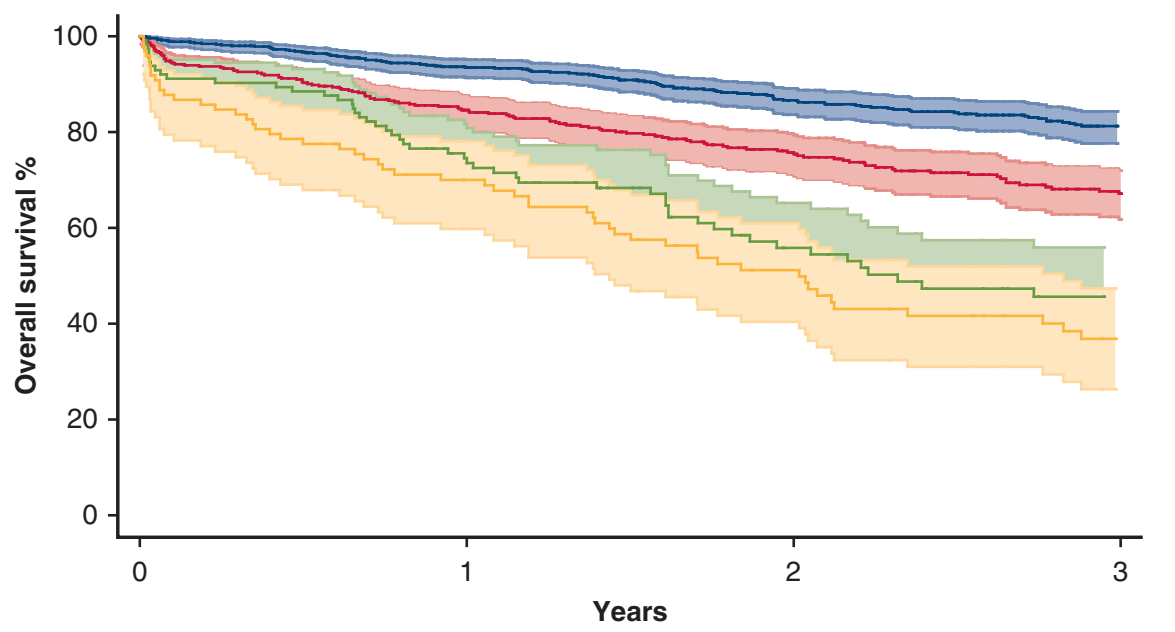

\begin{tabular}{|c|c|c|c|c|}
\hline \multicolumn{5}{|c|}{ Number at risk } \\
\hline - Class A & 702 & 578 & 421 & 294 \\
\hline —Class B & 445 & 340 & 227 & 139 \\
\hline - Class C & 113 & 73 & 41 & 25 \\
\hline _Class D & 98 & 62 & 39 & 21 \\
\hline
\end{tabular}

FIGURE 1. Kaplan-Meier OS estimates after lung cancer resection stratified by Eurolung classes of risk. Shaded areas represent $95 \%$ CIs. Higher Eurolung risk categories are associated with worse prognosis (log-rank test $P<.0001$ ). 


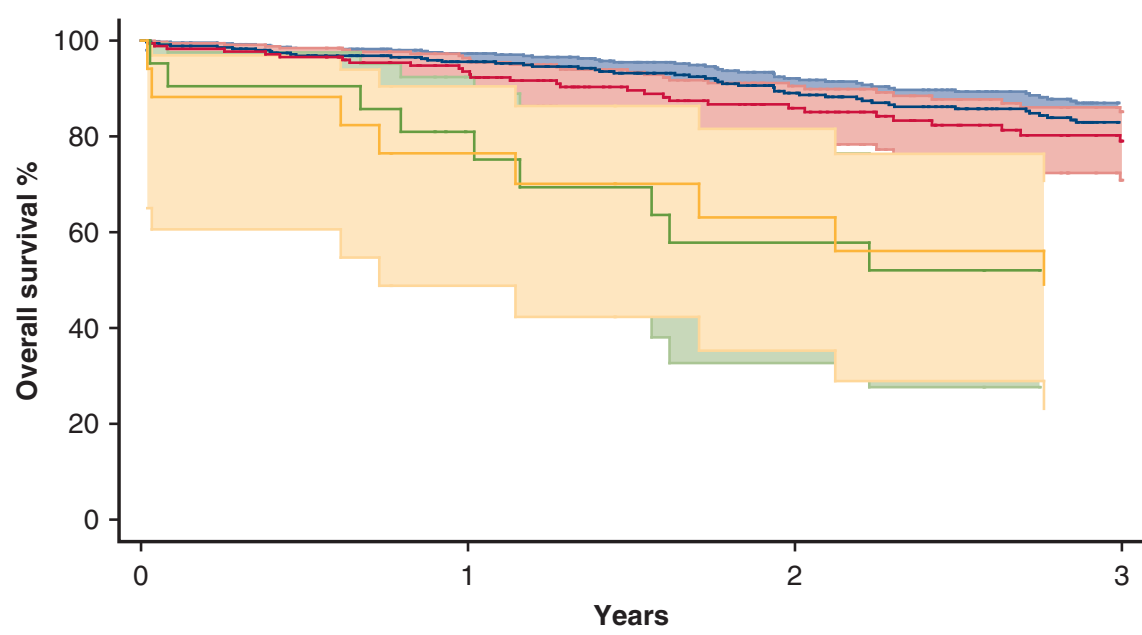

Number at risk

$\begin{array}{ll}\text { - Class A } & 352 \\ \text { - Class B } & 174 \\ \text { Class C } & 21 \\ \text { Class D } & 17\end{array}$

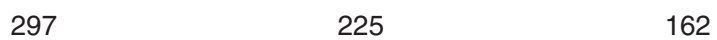

$\begin{array}{lll}151 & 107 & 65\end{array}$

$14 \quad 10$

A

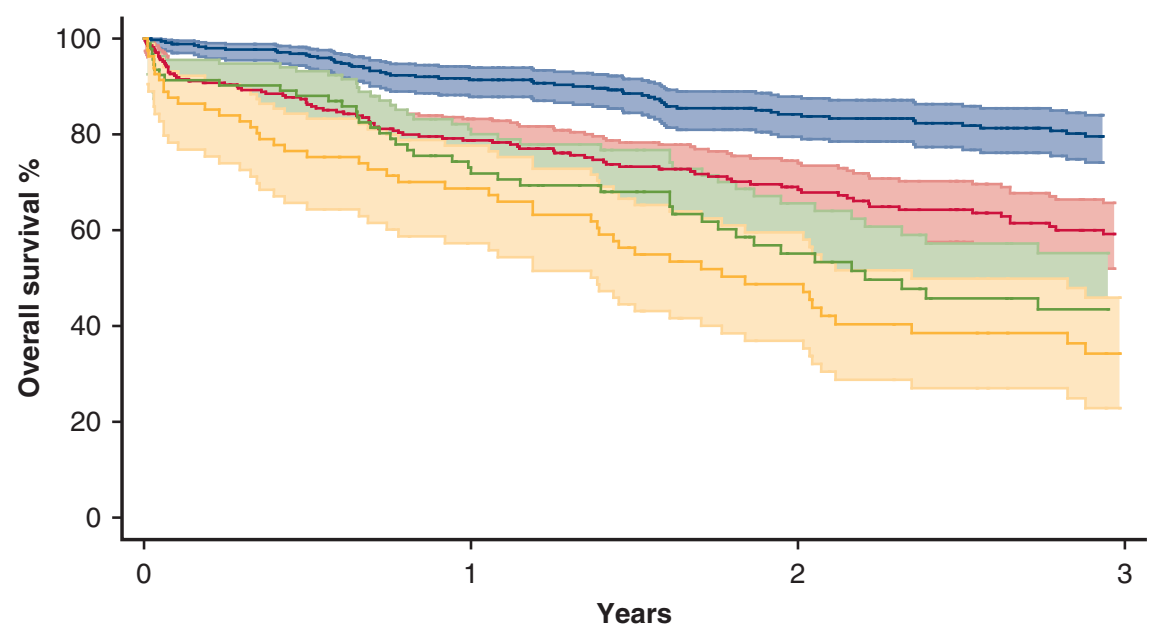

Number at risk

$\begin{array}{lr}\text { - Class A } & 350 \\ \text { - Class B } & 271 \\ \text { - Class C } & 92 \\ \text { - Class D } & 81 \\ \text { B } & \end{array}$

$281 \quad 196 \quad 132$

$189-120 \quad 74$

$59-31 \quad 18$

$50 \quad 30 \quad 14$

FIGURE 2. Kaplan-Meier OS estimates after lung cancer resection stratified by Eurolung risk classes showing a worse survival in higher Eurolung classes of risk either in patients with pT 1 stage, $P<.0001$ (A) and in those with pT greater than 1 stage, $P<.0001$ (B). Shaded areas represent $95 \%$ CIs.

patients with negative nodal disease $(P<.0001)$ and in those with positive nodes $(P=.0007)$ (Figure $3, A$ and $B$ ).

Patients with positive lymph nodes and in class A had a 3 -year OS of $65 \%$ (95\% CI, 52-75), whereas patients in class D had 39\% 5-year OS (95\% CI, 26-52). Patients with the most unfavorable scenario $(\mathrm{pT}$ stage greater than 1 and positive lymph nodes) showed 55\% 3-year OS in class A $(95 \%$ CI, 38-70), 53\% in class B $(95 \%$ CI, 37-67), 33\% in class C (95\% CI, 17-50), and $37 \%$ in class D $(95 \% \mathrm{CI}, 23-51)$.

On the other hand, patients with pT1N0 stage in class $\mathrm{D}$ had 51\% 3-year survival (95\% CI, 12-81), whereas 


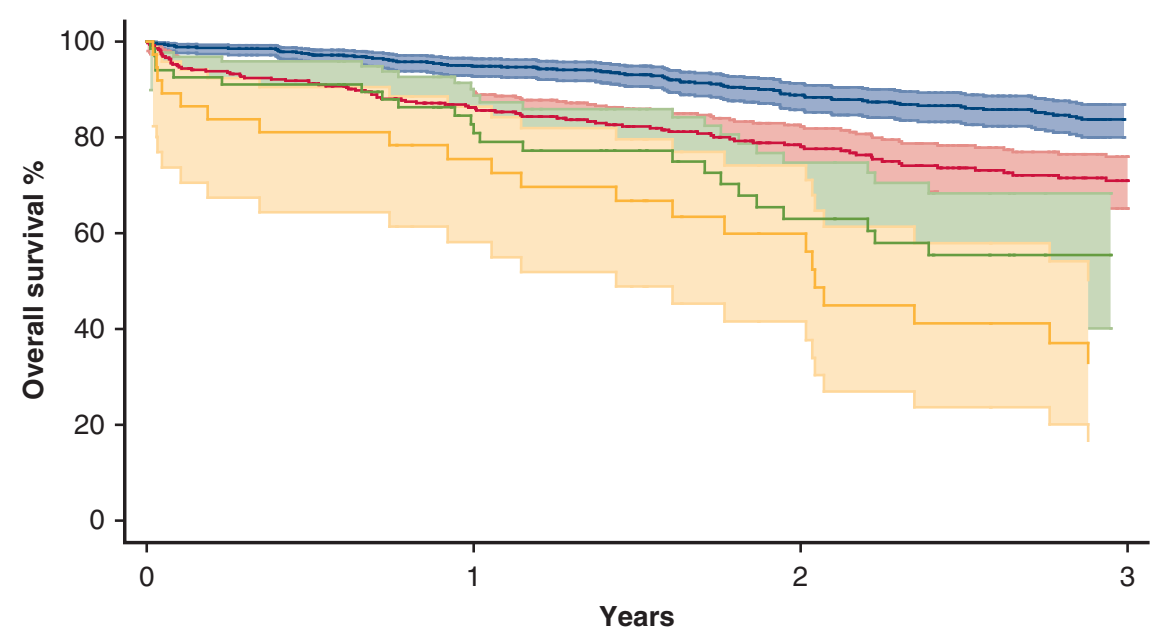

Number at risk

$\begin{array}{rcccc}- \text { Class A } & 610 & 511 & 374 & 266 \\ - \text { Class B } & 356 & 280 & 189 & 119 \\ \text { - Class C } & 67 & 45 & 26 & 16 \\ \text { - Class D } & 37 & 26 & 16 & 8\end{array}$

A

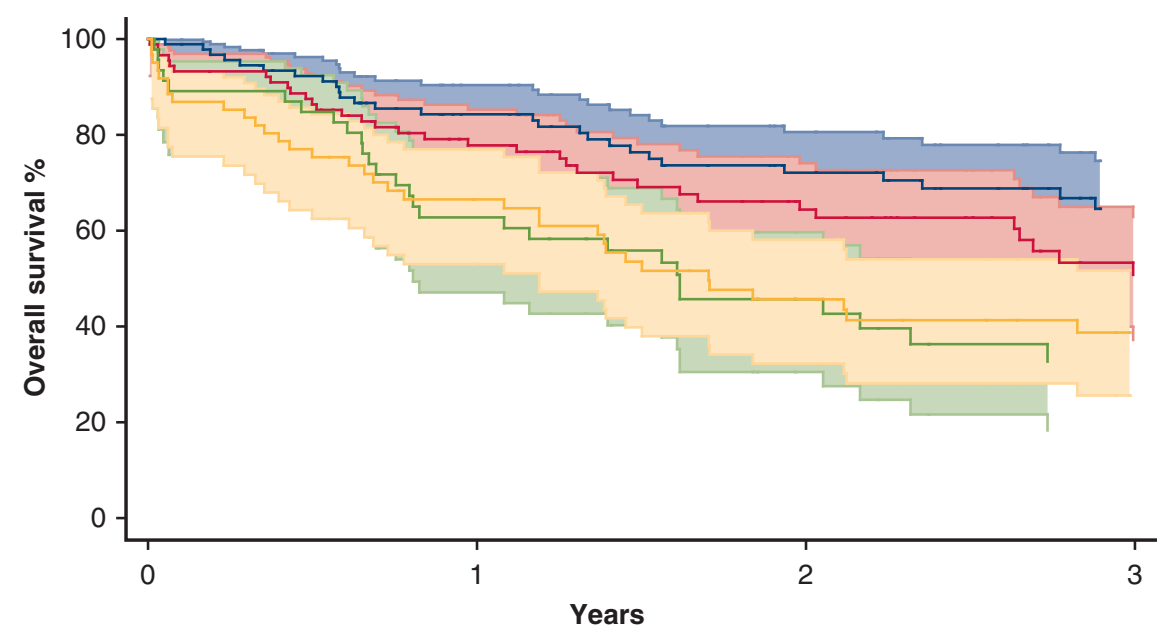

Number at risk

$\begin{array}{ccccc}\text { - Class A } & 92 & 67 & 47 & 28 \\ \text { - Class B } & 89 & 60 & 38 & 20 \\ \text { - Class C } & 46 & 28 & 15 & 9 \\ \text { - Class D } & 61 & 36 & 23 & 13\end{array}$

B

FIGURE 3. Kaplan-Meier OS estimates after lung cancer resection stratified by Eurolung risk classes showing a worse survival in higher Eurolung classes of risk either in patients with pN0 stage, $P<.0001$ (A) and in those with $\mathrm{pN}$ positive stage, $P=.0005$ (B). Shaded areas represent $95 \%$ CIs.

those in class $\mathrm{A}, \mathrm{B}$, and $\mathrm{C}$ had $84 \%$ (95\% CI, 78-88), $85 \%$ (795\% CI, 8-91), and 67\% (95\% CI, 33-86), respectively.

A Cox proportional hazard regression to adjust for $\mathrm{pT}$ and pN stage and other patient-related factors showed that Eurolung aggregate score as a whole covariate remained significantly associated with 3 year OS (hazard ratio, 1.2; 95\% CI, 1.1-1.2; $P<.0001$, Harrel's C concordance statistics 0.70) (Table 2).

A competing regression analysis (where the competing events were death from cancer other than lung cancer or death from causes other than cancer) confirmed the 
TABLE 2. Results of the Cox hazard regression analysis to verify the independent association of Eurolung aggregate score with 3-year overall survival

\begin{tabular}{lcllc}
\hline \multicolumn{1}{c}{ Variables } & HR & SE & $P$ value & $\mathbf{9 5} \%$ CI \\
\hline Eurolung class & & & & \\
$\quad$ Class A (no. 702) & Reference & & & \\
Class B (no. 446) & 1.8 & 0.2 & $<.0001$ & $1.4-2.4$ \\
Class C (no. 113) & 2.5 & 0.5 & $<.0001$ & $1.8-3.6$ \\
Class D (no. 98) & 2.6 & 0.5 & $<.0001$ & $1.8-3.8$ \\
ppoDLCO & 0.98 & 0.003 & $<.0001$ & $0.98-0.99$ \\
pT $>1$ & 1.6 & 0.2 & $<.0001$ & $1.3-2.1$ \\
pN positive & 1.7 & 0.2 & $<.0001$ & $1.4-2.2$ \\
\hline
\end{tabular}

Eurolung levels have been fitted as separate covariates. Only independent predictors with $P<.1$ are displayed. Other variables used in the regression analysis: coronary artery disease, cerebrovascular disease, chronic kidney disease, performance score, and diabetes. $H R$, Hazard ratio; $S E$, standard error; $C I$, confidence Interval; ppoDLCO, predicted postoperative carbon monoxide lung diffusion capacity.

independent association of the Eurolung score as whole covariate with 3-year DSS adjusting for $\mathrm{pT}$ and $\mathrm{pN}$ status and other patient-related factors (hazard ratio, $1.1 ; 95 \% \mathrm{CI}$, 1.03-1.18; $P=.006$ ) (Table 3).

Figure 4 shows the cumulative incidence of lung cancer-specific death in patients with different Eurolung categories. There is a progressively increasing risk of lung cancer death by Eurolung class of risk.

The score stratified the 3-year DSS in patients with pT greater than $1(P<.0001)$, with negative nodal disease $(P=.0001)$ and in those with positive nodes $(P=.019)$. In patients with $\mathrm{pT} 1$ disease, the different classes of Eurolung aggregate score were not associated with different DSS $(P=.29)$.

Patients with positive lymph nodes and in class A had a 3 -year DSS of $74 \%$ (95\% CI, 61-84), whereas patients in class D had 60\% 3-year DSS (95\% CI, 42-74). Patients

TABLE 3. Results of the competing regression analysis to verify the independent association of Eurolung aggregate score with 3-year disease-specific survival (competing risk: death from other cancers or death from noncancer causes)

\begin{tabular}{lcccr}
\hline \multicolumn{1}{c}{ Variables } & Cause-specific HR & SE & $\boldsymbol{P}$ value & $\mathbf{9 5 \%}$ CI \\
\hline Eurolung class & & & & \\
Class A (no. 702) & Reference & & & \\
Class B (no. 446) & 1.4 & 0.3 & .08 & $0.95-2.2$ \\
Class C (no. 113) & 2.4 & 0.6 & .001 & $1.4-4.0$ \\
Class D (no. 98) & 1.6 & 0.5 & .15 & $0.9-2.9$ \\
PS $>1$ & 1.6 & 0.4 & .049 & $1.0-2.6$ \\
pT $>1$ & 1.8 & 0.4 & .004 & $1.2-2.6$ \\
pN positive & 2.7 & 0.5 & $<.0001$ & $1.9-4.0$ \\
\hline
\end{tabular}

Eurolung levels have been fitted as separate covariates. Only independent predictors with $P<.1$ are displayed. Other variables used in the regression analysis: coronary artery disease, cerebrovascular disease, chronic kidney disease, diabetes, ppoDLCO. $H R$, Hazard ratio; $S E$, standard error; $C I$, confidence interval; $P S$, performance score. with negative nodal disease in class D had a 3-year DSS of $71 \%$ (95\% CI, 42-88) compared with patients in class A who had a DSS of $93 \%$ at 3 years (95\% CI, 90-95).

\section{DISCUSSION \\ Background and Rationale}

The Eurolung score was developed to stratify the risk of postoperative 30-day morbidity and mortality. ${ }^{1}$ The model includes several patient-related and surgicalrelated variables such as age, gender, ppoFEV1, BMI, extent of resection, and surgical access. Some of these factors are clearly associated with patient frailty and comorbidities, and others reflect the complexity of the operation often dictated by the locally advanced stage of disease. In addition, the extent of surgery may lead to further disabilities leading in turn to increased longterm morbidity and mortality. For this reason, we hypothesized that a cumulative score such as Eurolung may be associated not only with short term outcome but also with long-term survival after curative surgery. The rationale is to develop an additional tool that can be used in combination with the most traditional oncologic prognostic factors (eg, stage of disease) to enhance the prediction of long-term mortality to more specifically tailor curative treatment to individual patients.

\section{Main Finding}

The main finding of this study was that the Eurolung aggregate score was associated with an incremental risk of long-term overall and lung cancer-specific mortality. Patients in class D had 64\% and 73\% 3- and 5-year overall mortality rate, which was 3 -fold higher than the one observed in class A (with the lowest risk).

The incremental risk of long-term death is in line with the incremental risk of perioperative mortality shown in the original study ${ }^{1}$ and found in this set of patients. This is in part explained by the fact Eurolung is composed by factors reflecting the general fitness and frailty of patients, which may have an impact not only on the short-term outcomes but also on the long-term survival. Older age, reduced pulmonary function, and lower BMI encapsulating the risk of sarcopenia, as well as being a potential marker of more advanced disease, are well-known adverse prognostic factors in patients with lung cancer. Even more interesting is the finding that Eurolung is associated with lung cancerspecific mortality. This may be explained by the fact Eurolung could represent a surrogate for more aggressive and advanced disease that in turn portend a worse prognosis. In fact, factors such as open surgery and pneumonectomy (as opposed to lesser resection), which both have high scores in the Eurolung calculation may reflect a locally advanced stage of disease necessitating more complex operation. However, the effect of these more complex or extended procedure itself may have a negative impact on 


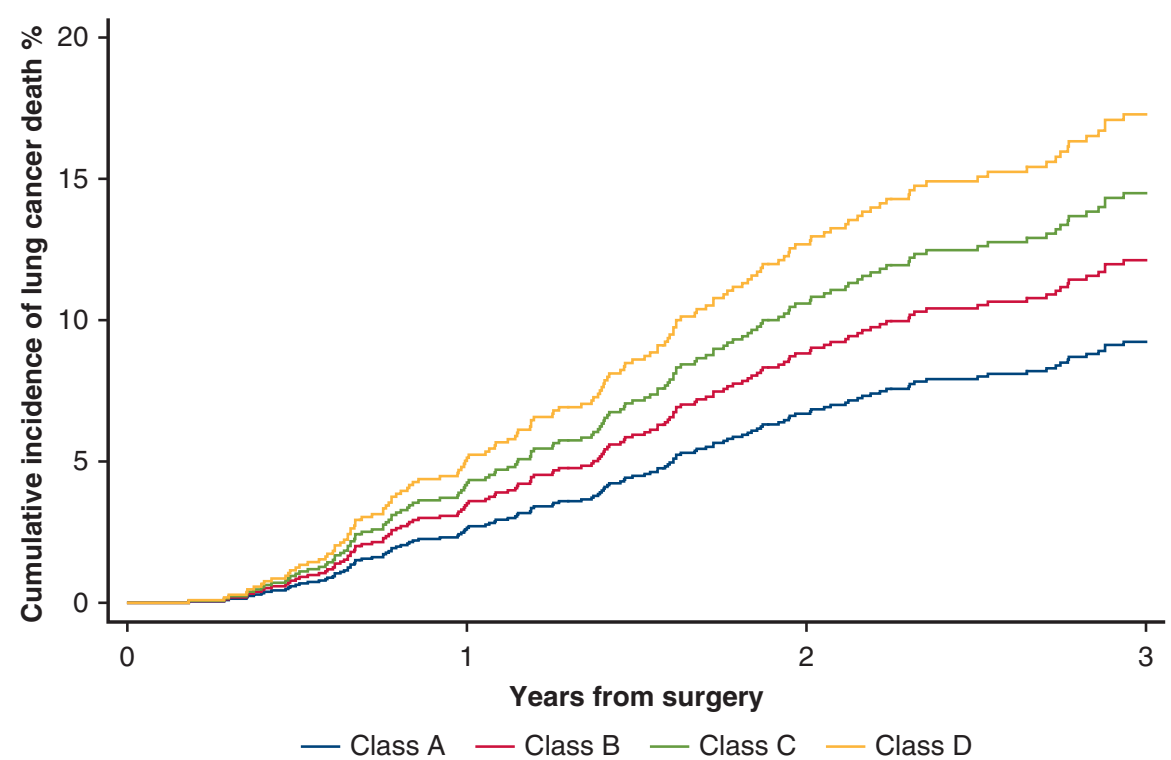

FIGURE 4. Cumulative incidence of lung cancer-specific death in patients with different Eurolung classes of risk. Competing regression analysis was used to adjust for $\mathrm{pT}$ and $\mathrm{pN}$ stage where the competing events were deaths for other cancers and for causes other than cancer.

prognosis leading to increased risk of deaths for cardiopulmonary complications. For instance, pneumonectomy has been associated with increased risk of long-term mortality. ${ }^{10-14}$ Additionally, physiologic variables may be associated with cancer progression. There is a cumulating body of evidence showing that better physical fitness is
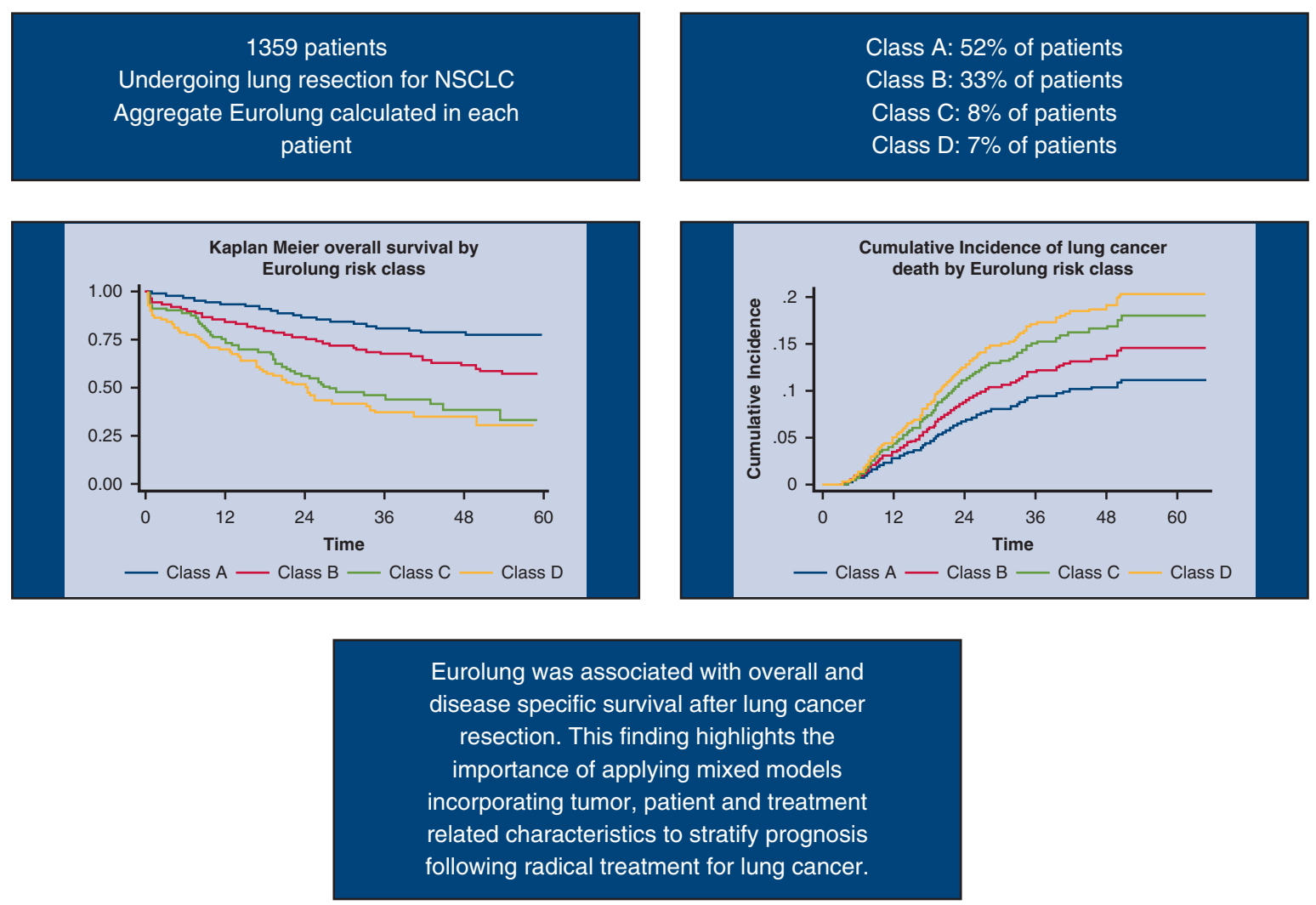

FIGURE 5. Summarizing the analyzed population and main findings of the analysis. NSCLC, Non-small cell lung cancer. 
associated with reduced risk of cancer-specific mortality and all-cause mortality. ${ }^{15-18}$

In patients with lung cancer, some markers of impaired physical condition and general deconditioning have been reported to be associated with long-term survival after surgery. ${ }^{2-6}$ Even the patient's perception of physical functioning and well-being has been found to be associated with overall and cancer specific survival after surgery. ${ }^{19,20}$

Better physical functioning and performance status may positively influence physiologic outcomes associated with cancer, such as a reduction in body weight and composition, and beneficial changes in metabolic and sex hormones, growth factors, adipokines, immune function, or inflammation that can potentially affect survival. ${ }^{21-23}$ In addition, a worse physical condition at the time of surgery may negatively affect the compliance with postoperative systemic treatments in more advanced stages of cancer (ie, adjuvant chemotherapy) leading to poorer outcomes.

In our study, one of the most important findings was the excellent survival of patients in the lowest class of risk despite their locally advanced stage of disease and the very poor prognosis of patients with early-stage disease in the highest class of risk. This highlights the importance of taking into account patient-related and treatment-related factors in addition of TNM staging to refine prognostic stratification after curative therapy.

The concept of using risk models including patient- and treatment-related variables to stratify the prognosis after lung cancer resection has been tested in previous studies. ${ }^{20,24-27}$ In general, these studies showed that these "mixed models" were able to stratify both the risk of allcause death and lung cancer-specific death, in line with our findings.

\section{Study Limitations}

The study may have potential limitations. First, the Eurolung was not specifically developed to predict long-term survival. The different factors compounding the score were originally weighed on their association with postoperative 30-day mortality. Unfortunately, the ESTS database does not collect long-term follow-up, preventing the development of a more specific score for survival. Although the use of a score to stratify prognosis is appealing, it must be interpreted with caution because it likely represents a surrogate measure of patient frailty and extent of disease. It would be desirable that more precise parameters or measures be developed and used in the future to improve prognostic stratification after curative treatment of lung cancer. The International Association for the Study of Lung Cancer has recently recognized this need and expanded their staging committee to include a prognostic subcommittee with the aim to identify methods and instruments to develop specific prognostic models incorporating patient, tumor, environment, and treatment-specific factors (https://www. iaslc.org/About-IASLC/IASLC-Committees). Although Eurolung has proven to be associated with OS and DSS, to be reliably used in the context of multifactorial prognostic instruments, it needs to be recalibrated to that specific outcome. Larger samples are needed to perform such recalibration and perhaps incorporate additional prognostic factors.

Generalization of the present findings to other centers require further confirmatory analyses because treatment variability may play an important role in long-term prognosis after curative resection for lung cancer. In addition, the 30-day mortality rate observed in the current series appears slightly higher than the one commonly reported in the most recent periods from international databases. This information needs to be accounted for when interpreting the results.

Eurolung incorporates variables related to the preoperative and operative phases. Postoperative factors influencing the physical and emotional status of the patient may also affect prognosis, but these are not captured in the Eurolung.

Only 98 patients were in the highest risk class. For this reason, all patients with a score of 7 or higher were collapsed into a single class (class D). That explains why in this study the aggregate Eurolung includes only 4 classes at variance with the original 7 classes. ${ }^{1}$ A larger sample size may allow a more granular analysis in the highest risk group of patients and refine their risk stratification.

\section{CONCLUSIONS}

The Eurolung aggregate score was associated with overall and DSS after curative resection for cancer. This association held after adjustment for pathologic $\mathrm{T}$ and $\mathrm{N}$ stages. The current analysis may represent valuable data to support and inform future studies on larger groups of patients aimed at developing specific multifactorial mixed prognostic models incorporating patient-related, treatment-related, and tumor-related factors to assist in refining the prognostic stratification in patient candidates with lung cancer to curative treatment (Figure 5).

Our findings may be valuable in the shared decisionmaking process when discussing with patients their treatment options allowing to manage their expectations with surgery. The score can inform on both short- and longterm outcomes. The score can inform the multidisciplinary discussion assisting in the selection of the most appropriate curative treatment in high-risk patients. As an example, during the period of maximum resource limitation imposed by the Coronavirus Disease 2019 pandemic, we have started to use this score in the multidisciplinary discussion to triage patients for surgery balancing surgical risk and surgical and intensive care capacity. 


\section{Webcast}

You can watch a Webcast of this AATS meeting presentation by going to: https://aats.blob.core.windows.net/media/ 20AM/Presentations/Eurolung \%20Risk \%20Score \%20is $\% 20$ Associated $\% 20$ wi.mp4.

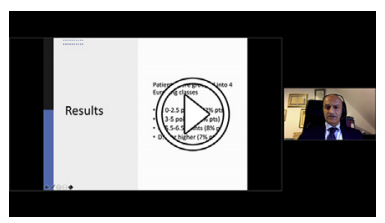

\section{Conflict of Interest Statement}

The authors reported no conflicts of interest.

The Journal policy requires editors and reviewers to disclose conflicts of interest and to decline handling or reviewing manuscripts for which they may have a conflict of interest. The editors and reviewers of this article have no conflicts of interest.

\section{References}

1. Brunelli A, Cicconi S, Decaluwe H, Szanto Z, Falcoz PE. Parsimonious Eurolung risk models to predict cardiopulmonary morbidity and mortality following anatomic lung resections: an updated analysis from the European Society of Thoracic Surgeons database. Eur J Cardiothorac Surg. 2020;57:455-61.

2. Jones LW, Watson D, Herndon JE 2nd, Eves ND, Haithcock BE, Loewen G, et al Peak oxygen consumption and long-term all-cause mortality in non-small cell lung cancer. Cancer. 2010;116:4825-32.

3. Brunelli A, Pompili C, Berardi R, Mazzanti P, Onofri A, Salati M, et al. Performance at preoperative stair-climbing test is associated with prognosis after pulmonary resection in stage I non-small cell lung cancer. Ann Thorac Surg. 2012; 93:1796-800.

4. Brunelli A, Pompili C, Salati M, Refai M, Berardi R, Mazzanti P, et al. Preoperative maximum oxygen consumption is associated with prognosis after pulmonary resection in stage I non-small cell lung cancer. Ann Thorac Surg. 2014; 98:238-42.

5. Ferguson MK, Watson S, Johnson E, Vigneswaran WT. Predicted postoperative lung function is associated with all cause long-term mortality after major lung resection for cancer. Eur J Cardiothorac Surg. 2014;45:660-4.

6. Ferguson MK, Dignam JJ, Siddique J, Vigneswaran WT, Celauro AD. Diffusing capacity predicts long-term survival after lung resection for cancer. Eur J Cardiothorac Surg. 2012;41:e81-6.

7. Brunelli A, Kim AW, Berger KI, Addrizzo-Harris DJ. Physiologic evaluation of the patient with lung cancer being considered for resectional surgery: diagnosis and management of lung cancer, 3rd ed: American College of Chest Physicians evidence-based clinical practice guidelines. Chest. 2013;143(5 Suppl): e166S-90S.

8. Fernandez FG, Falcoz PE, Kozower BD, Salati M, Wright CD, Brunelli A. The Society of Thoracic Surgeons and the European Society of Thoracic Surgeons general thoracic surgery databases: joint standardization of variable definitions and terminology. Ann Thorac Surg. 2015;99:368-76.

9. Fine JP, Gray RJ. A proportional hazards model for the subdistribution of competing risk. J Am Stat Assoc. 1999;94:496-509.

10. Alexiou C, Beggs D, Onyeaka P, Kotidis K, Ghosh S, Beggs L, et al. Pneumonectomy for stage I (T1N0 and T2N0) nonsmall cell lung cancer has potent, adverse impact on survival. Ann Thorac Surg. 2003;76:1023-8.

11. Pagès PB, Mordant P, Renaud S, Brouchet L, Thomas PA, Dahan M, et al. Sleeve lobectomy may provide better outcomes than pneumonectomy for non-small cell lung cancer. A decade in a nationwide study. J Thorac Cardiovasc Surg. 2017; 153:184-95.

12. Thomas P, Doddoli C, Thirion X, Ghez O, Payan-Defais MJ, Giudicelli R, et al. Stage I non-small cell lung cancer: a pragmatic approach to prognosis after complete resection. Ann Thorac Surg. 2002;73:1065-70.
13. Kim DJ, Lee JG, Lee CY, Park IK, Chung KY. Long-term survival following pneumonectomy for non-small cell lung cancer: clinical implications for follow-up care. Chest. 2007;132:178-84.

14. Simón C, Moreno N, Peñalver R, González G, Alvarez-Fernández E, GonzálezAragoneses F, Bronchogenic Carcinoma Cooperative Group of the Spanish Society of Pneumology and Thoracic Surgery. The side of pneumonectomy influences long-term survival in stage I and II non-small cell lung cancer. Ann Thorac Surg. 2007;84:952-8.

15. Holick CN, Newcomb PA, Trentham-Dietz A, Titus-Ernstoff L, Bersch AJ, Stampfer MJ, et al. Physical activity and survival after diagnosis of invasive breast cancer. Cancer Epidemiol Biomarkers Prev. 2008;17:379-86.

16. Irwin ML, Smith AW, McTiernan A, Ballard-Barbash R, Cronin K, Gilliland FD, et al. Influence of pre and post diagnosis physical activity on mortality in breast cancer survivors: the health, eating, activity, and lifestyle study. J Clin Oncol. 2008;26:3958-64.

17. Meyerhardt JA, Heseltine D, Niedzwiecki D, Hollis D, Saltz LB, Mayer RJ, et al. Impact of physical activity on cancer recurrence and survival in patients with stage III colon cancer: findings from CALGB 89803. J Clin Oncol. 2006;24:3535-41.

18. Holmes MD, Chen WY, Feskanich D, Kroenke CH, Colditz GA. Physical activity and survival after breast cancer diagnosis. JAMA. 2005;293:2479-86.

19. Pompili C, Salati M, Refai M, Berardi R, Onofri A, Mazzanti P, et al. Preoperative quality of life predicts survival following pulmonary resection in stage I nonsmall-cell lung cancer. Eur J Cardiothorac Surg. 2013;43:905-10.

20. Brunelli A, Salati M, Refai M, Xiumé F, Berardi R, Mazzanti P, et al. Development of a patient centered aggregate score to predict survival after lung resection for non-small cell lung cancer. J Thorac Cardiovasc Surg. 2013;146:385-90.

21. Ingram C, Courneya KS, Kingston D. The effects of exercise on body weight and composition in breast cancer survivors: an integrative systematic review. Oncol Nurs Forum. 2006;33:937-47.

22. Chlebowski RT, Aiello E, McTiernan A. Weight loss in breast cancer patient management. J Clin Oncol. 2002;20:1128-43.

23. McTiernan A, Ulrich C, Slate S, Potter J. Physical activity and cancer etiology: associations and mechanisms. Cancer Causes Control. 1998;9:487-509.

24. Balata H, Foden P, Edwards T, Chaturvedi A, Elshafi M, Tempowski A, et al. Predicting survival following surgical resection of lung cancer using clinical and pathological variables: the development and validation of the LNC-PATH score. Lung Cancer. 2018;125:29-34.

25. Lin J, Carter CA, McGlynn KA, Zahm SH, Nations JA, Anderson WF, et al. A prognostic model to predict mortality among non-small-cell lung cancer patients in the U.S. Military Health System. J Thorac Oncol. 2015;10:1694-702.

26. Tan HX, Drake BC, Chaudhuri N, Kefaloyannis M, Milton R, Papagiannopoulos K, et al. A risk model to predict 2-year survival after VATS lobectomy for non-small-cell lung cancer. Eur J Cardiothorac Surg. 2020;57:781-7.

27. Brunelli A, Ferguson MK, Salati M, Vigneswaran WT, Jimenez MF, Varela G. Thoracic revised cardiac risk index is associated with prognosis after resection for stage I lung cancer. Ann Thorac Surg. 2015;100:195-200.

Key Words: lung cancer, surgery, risk model, Eurolung, survival, risk stratification

\section{Discussion}

\section{Presenter: Dr Alessandro Brunelli}

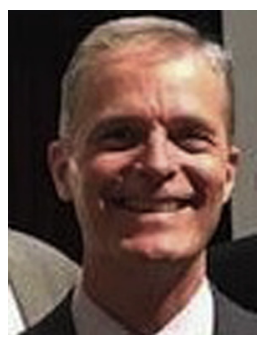

Dr John A. Howington (Nashville, Tenn). Your findings of worse outcomes with pneumonectomy and low BMI add to the other bodies of evidence showing poor outcomes in these groups. The findings of worse longterm outcome with open thoracotomy approach and male sex are thought provoking. As an example, a 71-year-old man with good lung 
function treated with an open thoracotomy lobectomy has a score of 6 and meets group 3 criteria, with profoundly reduced long-term survival independent of pathologic $\mathrm{T}$ stage and independent of node negative status or pT1. We know female sex portends worse outcome in cardiac surgery procedures and many critical care trials. To what do you attribute the dichotomy of significantly worse short-term and long-term outcomes in men with lung cancer treated with surgical resection?

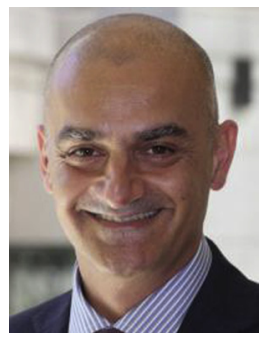

Dr Alessandro Brunelli (Leeds, UK). This is an important point. I would like to point out that we didn't want to recalibrate Eurolung on our population; we didn't isolate the individual factors compounding the Eurolung. So we don't really know whether these individual factors taken in isolation are associated with long-term prognosis as they are with perioperative mortality.

The simple reason why we didn't do this is because we think we don't have the sufficient number of events and the dataset to recalibrate the score on a long-term survival. What I can say for male sex, for instance, there have been some reports associating male sex with poor prognosis and they explain this in part with cultural reasons, especially in settings where the screening programs are not implemented; perhaps male patients tend to present at a later stage with more advanced disease. Also, it is well-known that male patients have a propensity for having severe comorbidities, such as ischemic heart disease, cerebrovascular disease, and other comorbidities that may have an impact on OS.

The other explanation I can think of is that perhaps the tumor in female patients may have more favorable histologic or genetic profiles that are associated with a more favorable prognosis in this gender.

Dr Howington. My next question relates to long-term outcomes. We are well aware of the data showing worse short-term outcomes with an open thoracotomy for lung cancer resection patients. However, your data will suggest a worse long-term outcome after a thoracotomy approach, despite matched pathologic stage of disease. To what do you attribute this worsened outcome? Can you tell from your data whether fewer patients after thoracotomy completed planned adjuvant chemotherapy or other interventions?

Dr Brunelli. It is difficult to draw conclusions on individual factors of the Eurolung. However, the thoracotomy may be a surrogate variable for a more complex operation in more advanced or locally advanced disease. We have stratified the pathologic $\mathrm{T}$ stage, but simply by dividing the group in $\mathrm{pT}=1$ or greater than 1 . We may have used a thoracotomy for a tumor larger than $5,6 \mathrm{~cm}$ or a tumor invading neighboring structures or for a sleeve resection.
All these operations performed for more locally advanced tumors are associated with a worse prognosis.

Regarding the adjuvant chemotherapy, obviously in a retrospective series, it is difficult to retrieve the exact indications to adjuvant chemotherapy and especially the reasons why patients didn't undergo adjuvant chemotherapy. We looked at those patients with the pathologic N1 and pathologic N2 disease, which is a more clear-cut indication for adjuvant chemotherapy. We didn't find any difference in terms of receiving chemotherapy after surgery between thoracotomy and minimally invasive surgery in our series.

Dr Howington. Have you altered your current approach to informed consent discussions with your patients? So when you're talking to a male patient who has moderate chronic obstructive pulmonary disease and may require an extended resection, is that altered-how you're having the discussion, and weighing, say, chemotherapy and radiation as an option versus surgical resection-particularly if it requires an open approach?

Dr Brunelli. The simple answer is yes. We have incorporated this score to provide more realistic and complete information to patients during consultation-and also during multidisciplinary discussion in tumor boards.

I think it is relevant to balance perioperative risk and long-term survival, especially in high-risk patients. This is the information that patients often ask about, at least in our setting, and what we wanted to develop was a readymade instrument that could inform the discussion. So yes, we are currently using this instrument to inform shared decision-making and informed consent.

Dr Howington. Again, thank you for an outstanding presentation and for adding to our knowledge and understanding about managing patients with lung cancer. And I appreciate the opportunity to be a discussant.

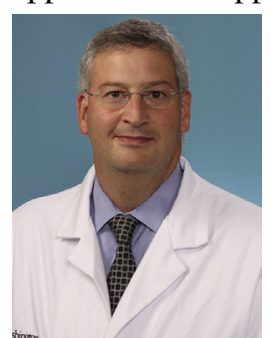

Dr Benjamin D. Kozower (St Louis, $M o$ ). A huge issue in the United States right now is data fatigue - the amount of data entry that data managers and faculty have to enter is incredible. So you have taken a very different approach. You have a model that is parsimonious using only 6 covariates to predict your outcomes. Do you recall what percent of the predictive capacity of the total model you have just using these 6 variables?

Dr Brunelli. The reason why we developed the parsimonious model is exactly the one you mentioned. The original Eurolung mortality model included 9 variables, so we thought that this was limiting the participation in the database and in the ESTS accreditation program. For this reason, we wanted to produce an easier, more user-friendly model without losing the predictive ability. In fact, the $\mathrm{C}$-indexes of the parsimonious morbidity and mortality models remained similar to the original ones. 
I believe the $\mathrm{C}$-index is approximately 0.78 . In any case, it is similar to the original model containing 9 variables. I appreciate by reducing the number of variables we may lose some information, especially in patients with cardiac risk, because there are no cardiac risk variables in the parsimonious model. However, with regard to the larger population-based risk prediction, the 2 models are similar.

Dr Kozower. That's great. With the current pandemic, there is great concern in the quality of data entry and kind of an opportunity for that. Are there any steps being taken by you and your team?

Dr Brunelli. Well, not specifically. We have a data manager now in our unit and in fact, her work is now simplified because we are operating fewer cases at the moment. We are at a very low capacity. We have reduced capacity from 11 surgical theaters a week to only 3 . This translates into perhaps 4 or 5 lung resections per week as a team. So, the data entry work is simplified in this regard. In relation to the Covid situation, there is a global registry collecting data on patients operated during this critical period and is UK-based (CovidSurg). We are inputting data in this registry to understand how the cancer pathways have been altered by the Covid situation-and this represents a double entry of data because it's a totally different registry from our institutional one. However, we think it is valuable to understand the changing practice we are undergoing at the moment.

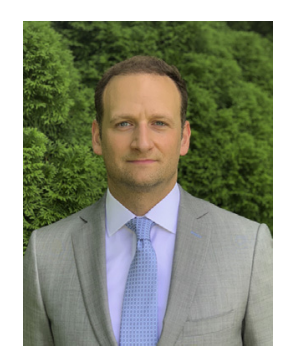

Dr Elliot L. Servais (Burlington, Mass). I did notice that you controlled in your regression analysis for pathologic $\mathrm{N}$ and $\mathrm{T}$ stage. What I didn't hear is whether you included sublobar resections in this analysis, and also whether there was any control for completeness of resection-maybe nodal stations harvested, number of nodes, and so forth.

Is there a possibility that somebody who is less fit with a worse Eurolung score undergoes a less aggressive resection, less lymph node harvest, maybe sublobar resection that could affect outcomes?

Dr Brunelli. Yes, we included segmentectomies. These are anatomic resections. We didn't include wedges. The segmentectomies are probably $5 \%, 7 \%$ of the total number of resections in our unit.

Unfortunately, there is no control in terms of number of lymph nodes because we don't collect that information in our database. We collect the type of lymphadenectomy based on the European definition. So systematic dissection versus lobar-specific sampling. Perhaps we can adjust these factors as well to ensure that we control, but at least there is a proper staging when we stratify by pathologic $\mathrm{N}$ status. Indeed, these are the data we have at hand. 